\title{
Broadband slot stripline antenna for high-precision positioning by signals of global navigational satellite systems
}

\author{
Vladimir Shepov ${ }^{1, *}$, Valery Vladimirov ${ }^{1,2}$, Alexander Kondratyev $^{2}$, and Vladimir Borisov ${ }^{2}$ \\ ${ }^{1}$ Reshetnev Siberian State University of Science and Technology, Krasnoyarsky Rabochy St., Kras- \\ noyarsk, 660037, Russia \\ ${ }^{2}$ Federal Research Center Krasnoyarsk Science Center of the Siberian Branch of the Russian Acade- \\ my of Sciences, Akademgorodok 50, Krasnoyarsk, 660036, Russia
}

\begin{abstract}
We present the results of the development of a new broadband slot stripline leaky wave antenna intended for high-precision positioning by signals of global navigation satellite systems (GNSS) in the following bands: GLONASS L1/L2/L3, GPS L1/L2/L5, GALILEO E5a/E5b/E6/E1, COMPASS B1/B2/B3.
\end{abstract}

\section{Introduction}

Broadband receiving antennas of high-precision positioning by signals of Global Navigataion Satellite Systems (GNSS) are widely represented on the global market, for example [1-3]. In addition, technical characteristics of antennas are steadily improved.

This paper briefly describes a new antenna intended for high-precision positioning by GNSS signals in the bands: GLONASS L1/L2/L3, GPS L1/L2/L5, GALILEO $\mathrm{E} 5 \mathrm{a} / \mathrm{E} 5 \mathrm{~b} / \mathrm{E} 6 / \mathrm{E} 1, \mathrm{COMPASS} \mathrm{B} 1 / \mathrm{B} 2 / \mathrm{B} 3$, as proposed in [4]. The slot stripline leaky-wave right-hand circular palarization antenna was used as a prototype. Antennas of the given type have a number of advantages: they are broadband with a wide radiation pattern, these antennas have small size and weight, and they are implemented on a single printed board substrate. The antennas have a stable phase center. Taking into account the offset of the antenna phase center (PCO) relative to its geometrical center and phase center variation (PCV) depending on the elevation angle $(\Theta)$ and azimuthal angle $(\varphi)$ of a received satellite signal is necessary in high-precision metrological GNSS applications using phase measurements.

The designed antenna is a low-profile one with a compact flat conducting ground plane. Thus, to fight the multipath effect main attention was paid to the characteristics of the antenna itself: radiation pattern, axial ratio, cross-polarization level and phase center stability.

\section{Antenna configuration and design}

Fig.1 presents an electrodynamical model of the designed broadband slot stripline leaky wave right-hand circular polarization antenna. The antenna is fabricated on a dielectric sub-

\footnotetext{
*Corresponding author: shepov@ksc.krasn.ru
} 
strate with two-side metallization (1). The thickness of the dielectric substrate is $1.524 \mathrm{~mm}$, with the diameter of $160 \mathrm{~mm}$ and permittivity $(\varepsilon)=3.5$.

Two slot radiators are implemented on the upper metal side of the dielectric substrate (2), which are intended for receiving GNSS signals. They are made in the form of spiral segments starting in the central part of the antenna and terminating at its edges. To receive a microwave signal with circular polarization the slots are coiled around the geometrical center of the antenna [5].

To improve the technical characteristics of this antenna it is suggested in [6] that additional slots (3) should be made between the main slot radiators of the antenna whose electrical length is several times shorter than the halfwavelength of the main slots at the highest operating frequency of the antenna. It is shown that additional slots implemented between the main slot radiators allows distributing microwave current antinodes more evenly along the whole length of the main slots. Owing to this effect the frequency dependence of the antenna gain becomes smooth, the axial ratio of the right-hand circular polarization signal is improved, and the phase center stability and cross-polarization suppression are increased [7-9].

A microstrip line (MSL) (4) is implemented on the lower metal side of the dielectric substrate. MSL is inductively coupled with the slots on the upper side of the substrate. To establish the leaky-wave mode, MSL is loaded with effective resistance equal to its characteristic impedance. A microwave signal is received by the slot radiators, then transmitted to MSL, to be further transferred to the feeder. To adjust the coefficient of inductive coupling of MSL with the slots the MSL characteristic impedance can be different from $50 \Omega$ and match with a $50-\Omega$ feeder by a wave-impedance transformer (5).

To suppress the back lobe of the antenna radiation pattern and multipath interference the simplest ground plane was used in the given antenna (6). The distance between the antenna and ground plane was optimized to steadily receive GNSS signals from $5^{\circ}$ above the horizon line, being equal to $12.7 \mathrm{~mm}$. Moreover, to suppress multipath effect and MSL radiation a microwave radiation absorber was implemented between the ground plane and radiator (7). The absorber dimensions were optimized in the following way: to decrease the cross-polarization the absorber width was increased. This was necessary in order to reduce the multipath effect on the accuracy characteristics of the GNSS receiver. Here, the gain for the right-hand circular polarization also decreased. This reduction was limited by the signal/noise ratio, which was necessary to steadily receive GNSS signals. The optimization criterion was the root-mean-square deviation (RMSD) of the relative positioning accuracy of antenna in the vertical and horizontal planes. As a result, for the microwave absorber with the insertion losses of $16 \mathrm{~dB} / \mathrm{cm}$ at $1 \mathrm{GHz}$ the outer radius of the circle was $72 \mathrm{~mm}$, and the circle width was $7.5 \mathrm{~mm}$.

A distinctive feature of the designed broadband antenna consists in the fact that the operating frequency bands of the antenna are generated by higher modes of the slots while MSL is implemented in the form of a double multimode microstrip leaky-wave resonator (MSR) [4].

The slots of different length, forming two subarrays. Fig. 1 shows the antenna prototype with twelve slots, six in each subarray. In one subarray the slot width is invariant and amounts to $300 \mu \mathrm{m}$. In the other subarray the slots are performed with a jump in the slot width, the minimum width being $250 \mu \mathrm{m}$, and the maximum - $450 \mu \mathrm{m}$. The distance from the inner end of the slots to the antenna center is adjusted, depending on the desired coefficient of inductive coupling of each slot with MSL and resonance frequencies adjusted to the desired frequency range.

By varying characteristic impedance in the appropriate segments of the slots it is possible to control their mode frequency [10]. This is necessary in order to generate each operating frequency band of the antenna by several higher modes of the slots of each subarray. As a result, the operating bandwidth of the antenna can be significantly enlarged. 
Fig. 2 (c) shows the designed MSL of the new antenna, (a) and (b) are the variants of the known MSL used in leaky-wave antennas intended for high-precision positioning by GNSS signals, (d) is the simulated coefficient $\mathrm{S}_{11}$ of the MSL data without taking into account slot radiators. For the matching of the MSL input impedance in a wider frequency range, MSL is designed so that the resonances of the second and third modes of MSR are within the frequency band of 1164-1300 (MHz). The resonance frequency of the fourth mode of MSR is adjusted to $1600 \mathrm{MHz}$ in order to receive microwave power from the slots within the range of 1559-1610 (MHz). As a result, the simulated length of the impedance transformer is $47.5 \mathrm{~mm}$, and the transformer width gradually decreases over $16.2 \mathrm{~mm}$ from $3.3 \mathrm{~mm}$ to $1.87 \mathrm{~mm}$. The width of each MSL at the junction with the transformer is $1.62 \mathrm{~mm}$, and further over $19.8 \mathrm{~mm}$ it smoothly decreases down to $0.8 \mathrm{~mm}$ and then, remains constant. The length of the outer MSL is equal to $176.8 \mathrm{~mm}$, and that of the inner MSL is $154.6 \mathrm{~mm}$. Both MSLs are loaded with effective resistance of $150 \Omega$.

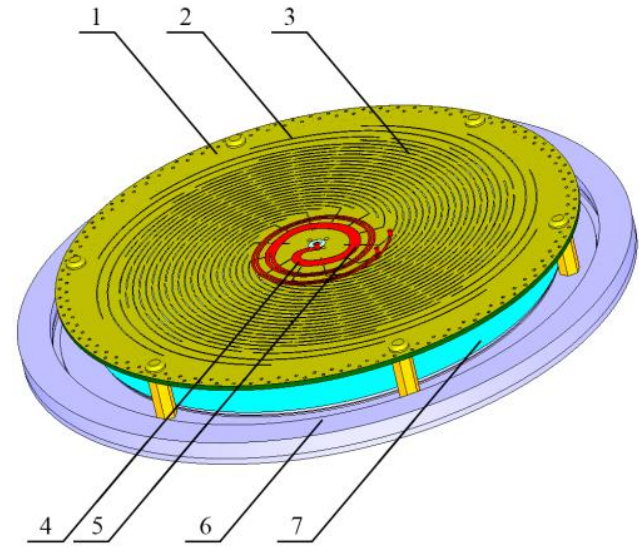

Fig. 1. Simulation model of the designed antenna. Fig. 2. (a) and (b) are the well-known MSL,

(c) MSL of the designed antenna, (d) Simulated input reflection coefficient.

\section{Results and discussion}

One can see in Fig. 2 (d) that the designed MSL allows adjusting resonance frequencies of higher modes of MSL so that the second and third modes of MSL receive signals from the slots within the range of 1164-1300 (MHz), and the fourth mode within 1559-1610 (MHz). Therefore, the input impedance of the antenna at a level $\mathrm{S}_{11}=-15 \mathrm{~dB}$ can be matched in a wider frequency range.

The simulated radiation pattern of the antenna at $1202 \mathrm{MHz}$ is presented in Fig. 3, where (1) is the radiation pattern for the right-hand circular polarization, and (2) is the radiation pattern for the left-hand one. The radiation pattern for the right-hand circular polarization is seen to be a wide-angled one with an abrupt decline. The gain reduction $(\mathrm{G})$ from zenith to horizon is equal to about $12 \mathrm{~dB}$, the suppression of the left-hand circular polarization amounts to about $-20 \mathrm{~dB}$ in zenith and $-22 \mathrm{~dB}$ at the elevation angles of $5^{\circ}$. At other operating frequencies of the antenna, radiation patterns are similar.

Fig. 4 shows the simulated frequency dependence of the antenn gain in the zenith radiation pattern. The antenna bandwidth of $1164-1300(\mathrm{MHz})$ is generated by three higher modes of the slot radiators, two of these modes resulting from one subarray and the third from another one. It is possible to bring closer the frequencies of two higher modes of one 
subarray owing to the performed jump of the characteristic impedance. The bandwidth of $1559-1610(\mathrm{MHz})$ is generated by two higher modes - one higher mode from each subarray.

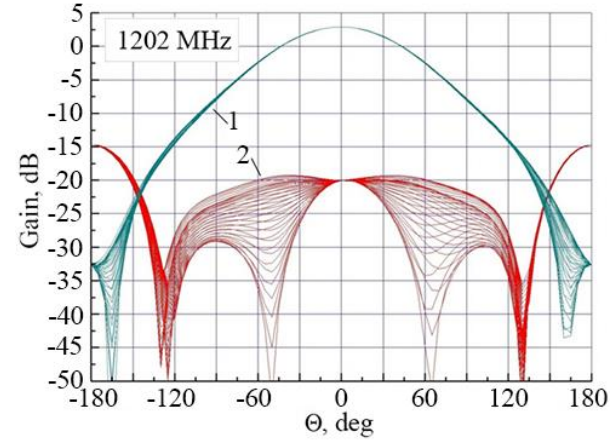

Fig. 3. Simulated radiation patterns.

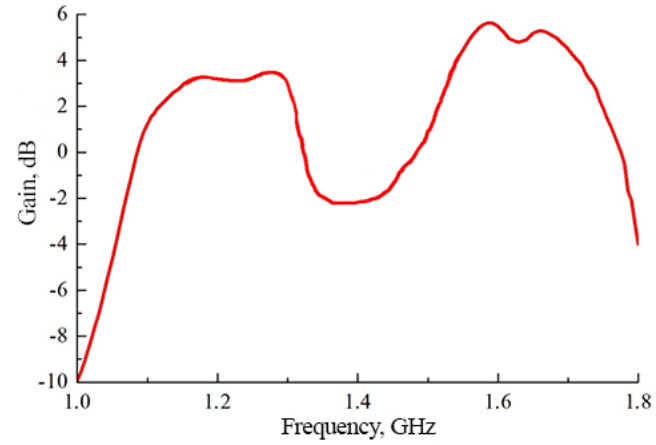

Fig. 4. Simulated gain of the proposed antenna.

The simulated dependences of the axial ratio (AR) of the antenna on the elevation angle $\Theta$ for the GNSS carrier frequencies at $\varphi=0$ are given in Fig. 5 (a). It can be seen in Fig. 5 (a) that AR does not exceed $1.5 \mathrm{~dB}$ at all the GNSS carrier frequencies in the zenith radiation pattern and is no more than $5 \mathrm{~dB}$ at the elevation angle of $5^{\circ}$.

The dependences of the antenna AR on the elevation angle $\Theta$ at $1246 \mathrm{MHz}$ when measuring $\varphi$ from 0 to $360^{\circ}$ are presented in Fig. 5 (b). One can see that for all $\varphi$ the axial ratio does not exceed $3 \mathrm{~dB}$ at $\Theta \leq 5^{\circ}$.

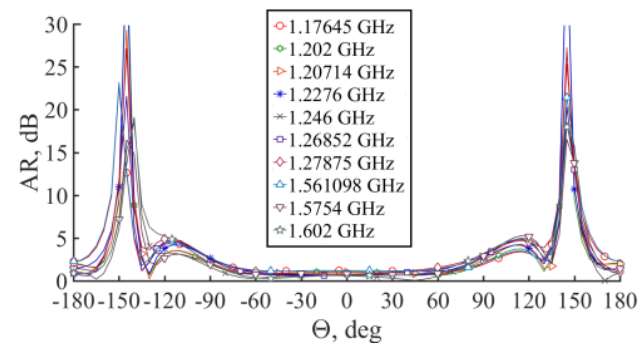

(a)

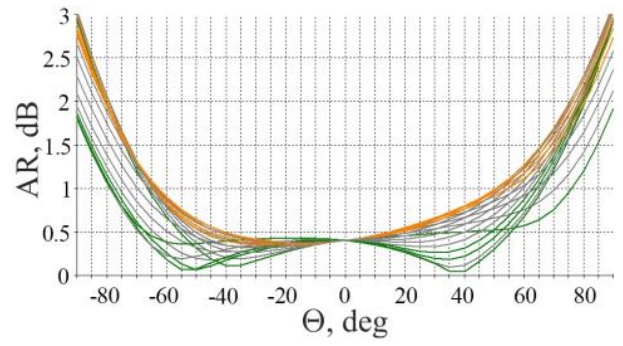

(b)

Fig. 5 Simulated AR patterns.

The offsets of the phase centers of the antenna relative to its geometrical center for the GNSS carrier frequencies are shown in Fig. 6 (a). The PCO coordinates are given in Table 1. As is seen in Table 1 , the simulated PCOs from the vertical axis do not exceed $2^{\circ}$. The exact coordinates of the phase centers are necessary for high-precision phase measurements.

Table 1. The PCO coordinates.

\begin{tabular}{|c|l|c|c|c|}
\hline$№$ & \multicolumn{1}{|c|}{$f, \mathrm{GHz}$} & $\varphi^{\circ}$ & $\Theta,^{\circ}$ & $R, \mathrm{~mm}$ \\
\hline 1 & 1.17645 & 286 & 2 & 13.36 \\
\hline 2 & 1.202 & 237 & 1 & 10.98 \\
\hline 3 & 1.20714 & 118 & 1 & 10.33 \\
\hline 4 & 1.22760 & 195 & 1 & 10.29 \\
\hline 5 & 1.246 & 166 & 1 & 10.30 \\
\hline 6 & 1.26852 & 133 & 1 & 10.36 \\
\hline 7 & 1.27875 & 228 & 1 & 10.74 \\
\hline 8 & 1.561098 & 232 & 1 & 5.80 \\
\hline 9 & 1.5754 & 247 & 1 & 4.01 \\
\hline 10 & 1.602 & 315 & 1 & 3.15 \\
\hline
\end{tabular}


Fig. 6 (b) presents the simulated PCV of the antenna at $1202 \mathrm{MHz}$. One can see that PCV is no more than $1.5 \mathrm{~mm}$ within the angles $\Theta= \pm 85^{\circ}$ and $\varphi= \pm 180^{\circ}$, where $\Theta=0$ is the zenith of radiation pattern. At other operating frequencies of the antenna the PCV dependences on $\Theta$ and $\varphi$ are similar.

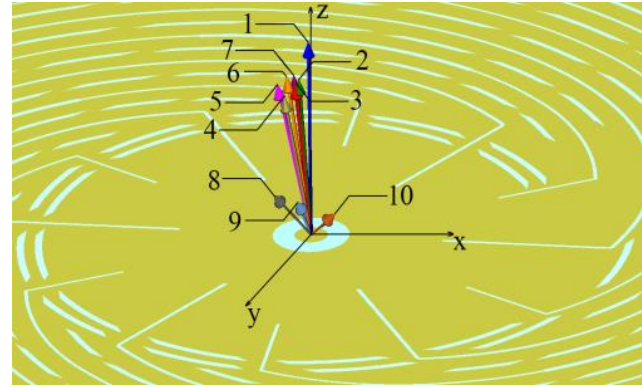

(a)

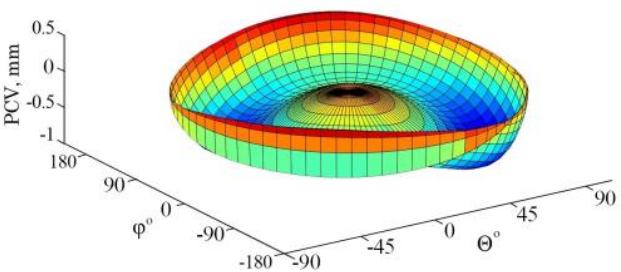

(b)

Fig. 6. (a) Graphic representation of the offsets of the antenna phase centers relative to its geometrical center, (b) graphic representation of the simulated PCV.

Figure 7 shows the photo of the proposed antenna, where (a) is the front view, and (b) is the back side of the antenna. The diameter of the antenna body is $185 \mathrm{~mm}$ and its height including the cover is $75 \mathrm{~mm}$.

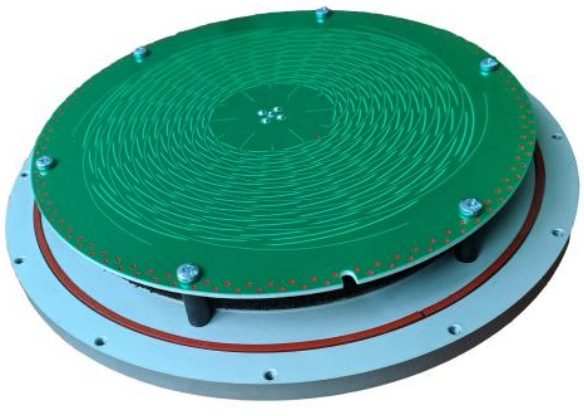

(a)

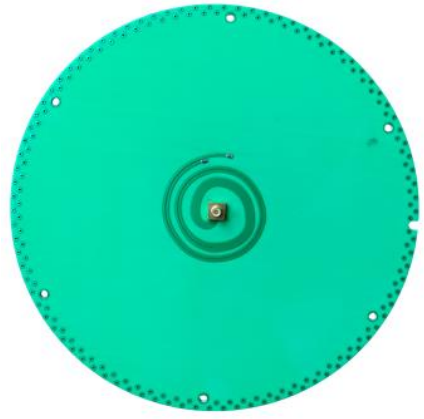

(b)

Fig. 7. Photo of the proposed antenna. (a) general layout of the active antenna. (b) back side of the antenna radiator.

The comparison of the simulated and measured reflection coefficient of the proposed antenna is given in Fig. 8. One can see that within the frequency range of 1164-1300 (MHz) and 1559-1610 (MHz) the measured values are $\mathrm{S}_{11}<-14 \mathrm{~dB}$.

Figure 9 shows the comparison of the normalized radiation patterns. The measured radiation pattern is seen to be in good agreement with the simulated one within the operating elevation angles of the antenna $\left( \pm 85^{\circ}\right.$ from zenith) and to have worse suppression of the back lobe, which can be due to incomplete insulation of a metal positioning mount in a anechoic chamber.

The comparison of the frequency dependences of the simulated and measured gain and axial ratio of the proposed antenna is presented in Fig. 10 and Fig. 11, respectively. The measured gain is $3 \pm 0.5(\mathrm{~dB})$ in the frequency range of $1164-1300(\mathrm{MHz})$ and $4.5 \pm 0.5(\mathrm{~dB})$ within the range of 1559-1610 (MHz). The measured AR is lower than $2 \mathrm{~dB}$ in all the operating frequency bands. 


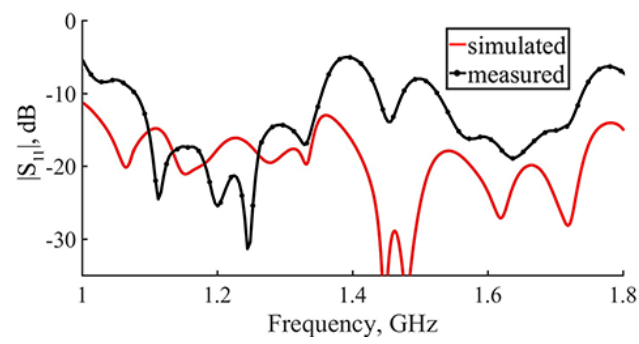

Fig. 8. Simulated and measured input reflection coefficient of the proposed antenna.

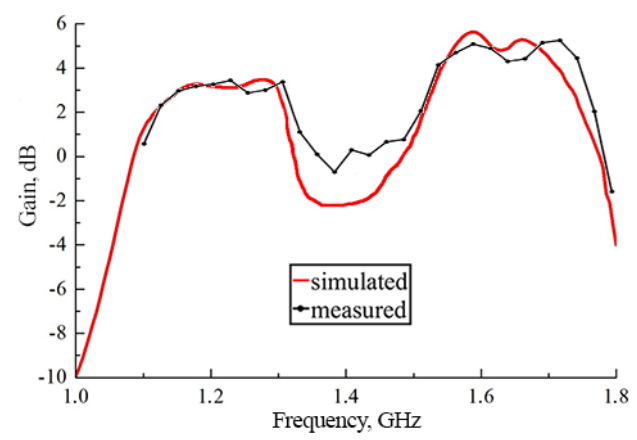

Fig. 10. Simulated and measured gain of the proposed antenna.

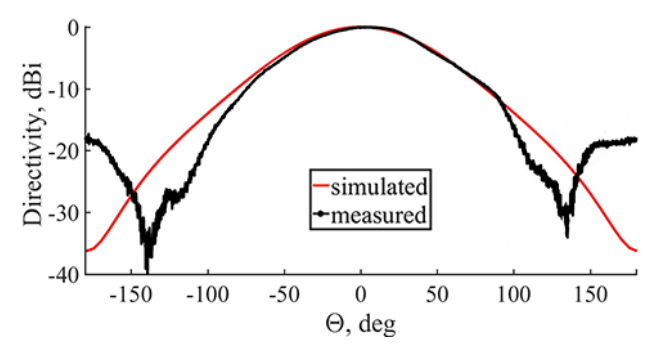

Fig. 9. Simulated and measured radiation patterns.

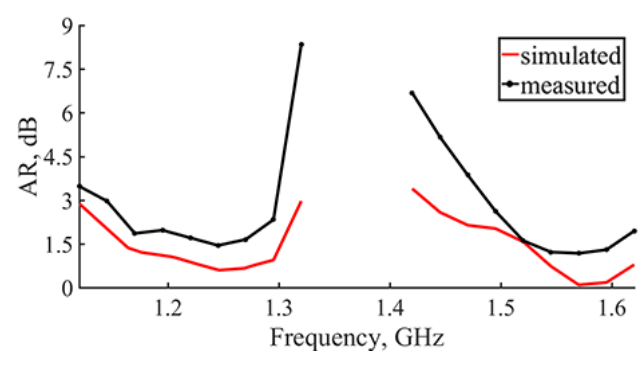

Fig. 11. Simulated and measured AR.

\section{Conclusion}

The paper presents a novel design of broadband slot stripline leaky wave right-hand circular polarization antenna. The application of a new design of MSL and method of generating operating frequency bands of the antenna by several higher modes of the slot radiators allows one to significantly extend the bandwidth of the antenna. The designed antenna receives navigation signals within the range of 1164-1300 (MHz) and 1559-1610 (MHz) from $5^{\circ}$ above the horizon line. The stable phase center, good axial ratio and rather high level of cross-polarization suppression allows using the presented antenna for high-precision GNSS applications involving phase measurements.

The antenna is fabricated on a single printed substrate. To suppress the back lobe of the radiation pattern and multipath interference a compact flat conducting ground plane is used in the antenna. The given advantages of the designed antenna allows fabricating it in a lowprofile variant to apply the antenna in spacecraft.

For the application of the given antenna in the fixed GNSS base stations it is possible to further suppress the multipath effect by using ground planes of special design, for example, a conventional choke ring ground plane and its modifications.

\section{References}

1. Source: http://www.leica.com/, Accessed: 22-07-2019.

2. Source: https://javad.com/, Accessed: 24-07-2019.

3. Source: https://www.trimble.com/, Accessed: 25-07-2019. 
4. V.N. Shepov, V.V. Markov Broadband slit stripline GNSS antenna, Patent RU No. 2619846 (2017)

5. W. Kunysz Aperture coupled slot array antenna, Pat. US 0067315Al. (2002)

6. V.M. Vladimirov, V.N. Shepov, Y.V. Krylov, and V.V. Markov A slot stripline leaky wave antenna with circular polarization, Patent RU No. 2504055 (2012)

7. V.M. Vladimirov, A.S. Kondratyev, Y.V. Krylov, et al., Rus. Phys. J., 55, No. 8, pp. 951-955 (2013)

8. V.M. Vladimirov, Yu.L. Fateyev, V.N. Shepov, Proc. of $23^{\text {rd }}$ International Crimean Conference Microwave \& Telecommunication Technology (CriMiCo'2013) Sevastopol, P. $594-595$ (2013)

9. V.N. Shepov, V.M. Vladimirov, V.V. Markov, Journal of Communications Technology and Electronics, 62, No. 7, pp. 770-779 (2017)

10. V.N. Shepov, V.V. Markov Circular polarisation slit stripline leaky-wave antenna with slit radiator width discontinuity, Patent RU No. 2580869 (2016) 